\title{
Guidelines for Understanding Magnification in the Modern Digital Microscope Era
}

\author{
James A. DeRose* and Michael Doppler
}

Leica Microsystems, Max Schmidheiny Strasse 201, Heerbrugg SG, CH-9435, Switzerland

*James.DeRose@leica-microsystems.com

\begin{abstract}
For digital light microscopes, which have image sensors but no eyepieces, the image is displayed directly on an electronic monitor. This development brings a significant change to the usual way magnification of an object viewed via a microscope is determined. Over the years, there have been various standards that define magnification when viewing an image through a microscope's eyepieces. Only recently have magnification standards been developed that apply to a digital microscope. This article offers guidelines for determining the range of useful magnification values for digital microscopy, particularly when monitors of various sizes are employed.
\end{abstract}

Keywords: digital light microscopy, magnification range, resolution, megapixel camera, field of view

\section{Introduction}

For more than 150 years light microscopy, employing glass lenses to focus light and produce the phenomenon of magnification, has allowed the observation of minuscule entities not visible to the unaided eye. Today there are many types of light microscopes, but here we discuss two of the most common: (1) digital microscopes employing electronic image sensors, but no eyepieces, where the image is displayed on an electronic monitor, and (2) microscopes for direct visual observation which have eyepieces and a digital camera, allowing it to be used in a similar manner to a digital microscope.

A feature of interest for any microscope is magnification. Image magnification is the apparent size of an object at a scale larger (or smaller) than its actual size. Magnification serves a useful purpose only when the resolution of the microscope makes it possible to see more details of an object in the image than when observing the object with the unaided eye. Until recently, magnification has been well defined when viewing an image of a sample through the eyepieces of a microscope. For this case, rigorous international standards have been documented [1-8]. Many of these standards also apply to digital microscopy, but strict definitions and standards for magnification achieved by a digital microscope, where the image is viewed on an electronic monitor, have been developed only in the last few years [9].

In research publications and presentations, images must include a calibrated scale bar or a statement of the width of the field of view, typically in $\mathrm{nm}$ or $\mu \mathrm{m}$. However, when viewing images in real-time on monitors of various sizes, indications of scale may not be shown, and the instantaneous magnification of an image may not be known. Moreover, there is typically a lower and higher limit of magnification which define the range of useful magnification. Digital microscopes, as well as microscopes for visual observation equipped with digital cameras, allow the rapid acquisition of high-quality images. These instruments are applied in various scientific and engineering fields.

The goal of this article is to provide digital microscopy users with helpful guidelines so they can determine the range of useful magnification when making observations and measurements. The specific parameter values given here are for instruments from Leica Microsystems, but the principles described are quite general for all light microscopes employing digital image acquisition and display.

\section{Magnification}

What exactly is magnification? Magnification is the ratio of the size of an object in an image produced by an optical system to the actual size of the object itself. Thus, lateral magnification, $M_{D I S}$, can be defined as:

$M_{D I S}=\frac{\text { dimension of an object in the image }}{\text { true dimension of the object }}$

Of course, the upper limit of visual magnification depends on the maximum resolving power of the microscope system. When the magnification is pushed above the useful range, no additional details about the sample can be seen. This situation is referred to as empty magnification [10]. Even within the
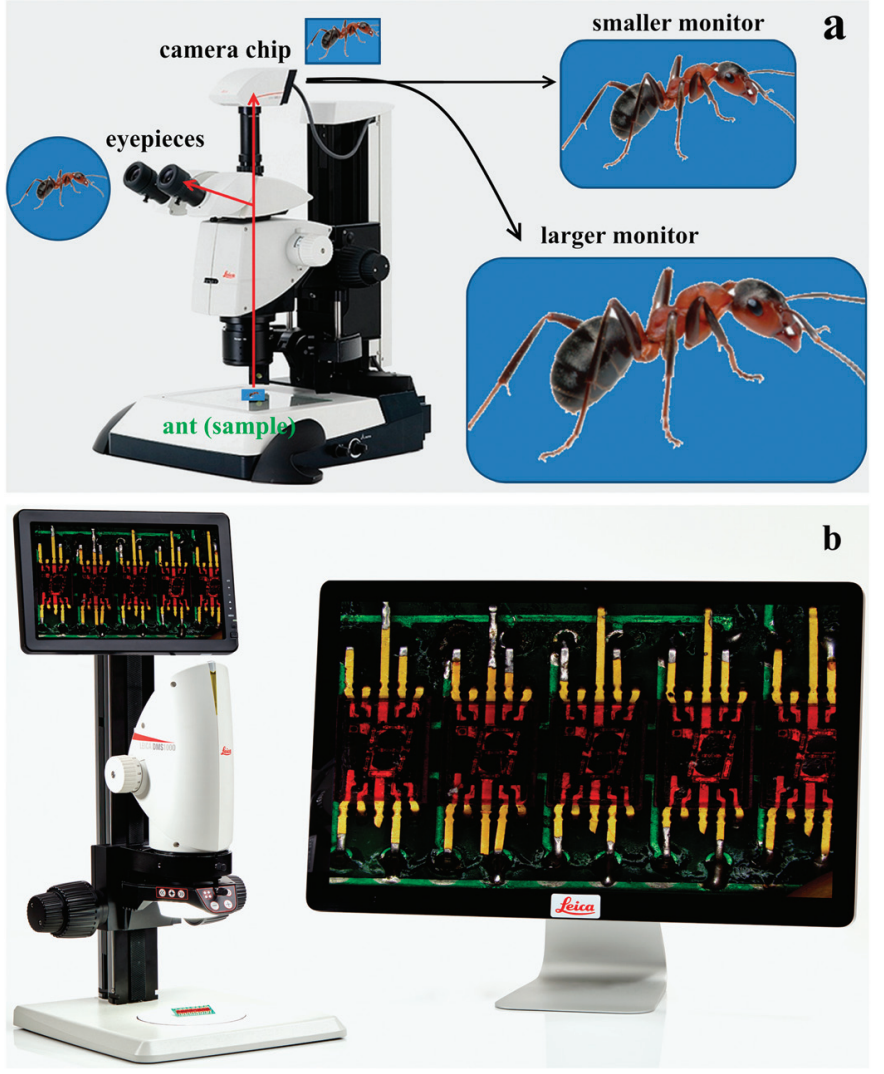

Figure 1: (a) Stereo microscope (M205 C) equipped with a digital camera. The ant sample can be observed via the eyepieces or on an electronic monitor displaying the image from the sensor in the camera. (b) Digital microscope (DMS1000) shown with two different monitor sizes for image display. 

microscope's resolving power, the practical level of observable detail also depends on the distance between the display of the image and the observer's eyes.

\section{Microscopes with Digital Sensors}

Magnification in conventional light microscopes. When observing the image through the eyepieces of a microscope for visual observation, the total magnification is defined as $[5,6]$ :

$M_{\text {TOT VIS }}=M_{O} \cdot q \cdot M_{E}$

where $M_{T O T}$ VIS is the total lateral magnification observed through the eyepiece, $M_{O}$ is the objective lens magnification, $q$ is the total tube factor (zoom and other tube lenses), and $M_{E}$ is the eyepiece lens magnification. As an example, a microscope with a $40 \times$ objective $\left(M_{O}=40 \times\right)$, total tube factor of $1 \times(q=1 \times)$, and $10 \times$ eyepieces $\left(M_{E}=10 \times\right)$, then the total magnification $\left(M_{\text {TOT VIS }}\right)$ seen via the eyepieces would be: $M_{\text {TOT VIS }}=40 \times \cdot 1 \times \cdot 10 \times=400 \times$.

Magnification at the digital sensor. For the case of a microscope image that is projected onto an electronic sensor, such as that of a digital camera, the magnification for the image formed at the sensor is $[5,6]$ :

$$
\begin{aligned}
& M_{\text {TOT PROJ }}=M_{O} \cdot q \cdot M_{E} \cdot p \quad \text { (projection via eyepiece) } \\
& M_{\text {TOT PROJ }}=M_{O} q \cdot M_{P H O T} \quad \text { (projection via tube) }
\end{aligned}
$$

where $M_{\text {TOT PROJ }}$ is the (lateral) magnification of the microscope (image projected onto the sensor), $p$ is the projection factor from eyepiece to camera, and $M_{P H O T}$ is the magnification of the photographic projection lens from tube to camera. Standard values for the total tube factor, $q$, fall between $0.5: 1$ and 25:1 [1]. Standard values for the photographic projection lens magnification, $M_{P H O T}$, fall between 0.32:1 and 1.6:1 [1].

Digital microscopes. For digital microscopes, there are no eyepieces, so an image is projected onto and detected by the electronic sensor and displayed on an electronic monitor for observation. The same is also true for a microscope for visual observation equipped with a digital camera when the image is observed via a monitor. Thus, the final total magnification for digital microscopy, $M_{D I S}$ (Equation 1), will always depend on the size of the image on the monitor versus the size on the sensor and can be defined as:

$$
M_{D I S}=M_{T O T} \text { PROJ } \cdot \text { image size ratio }
$$

where $M_{D I S}$ is the total lateral display magnification for an image displayed on a monitor, and the image size ratio is the "enlargement" of the image from the sensor to the electronic monitor display. As an example, if a microscope has a $20 \times$ objective $\left(M_{O}=20 \times\right)$, total tube factor of one $(q=1)$, a $0.5 \times$ photographic projection lens $\left(M_{P H O T}=0.5 \times\right)$, and a sensor and monitor with an image size ratio of 100:1, then the total display magnification $\left(M_{D I S}\right)$ seen via the image displayed on the monitor would be: $M_{D I S}=20 \times \cdot 1 \times \cdot 0.5 \times \cdot 100=1,000 \times$.

For an image size ratio, just one dimension could be used, such as the image width. The size of the image width on the monitor equals the number of monitor pixels in the image width times the pixel size. The same type of argument applies for the image width on the sensor:

$M_{\text {DIS }}=M_{\text {TOT PROJ }}$.

number of pixels for monitor image width $\cdot$ monitor pixel size number of pixels for sensor image width $\cdot$ sensor pixel size

The ratio of the monitor to sensor pixel size can be defined as the pixel size ratio:

$M_{\text {DIS }}=M_{\text {TOT PROJ }}$.

number of pixels for monitor image width number of pixels for sensor image width $\cdot$ pixel size ratio

When the number of monitor and sensor pixels are the same (a 1-to-1 pixel correspondence), then:

$M_{D I S}=M_{T O T \text { PROJ }} \cdot$ pixel size ratio

As just stated, the pixel size ratio is the ratio of the pixel size of the monitor to that of the sensor:

pixel size ratio $=\frac{\text { monitor pixel size }}{\text { sensor pixel size }}$

For Equation 5, it is assumed that the number of pixels across the image on both the sensor and the monitor occur in a 1-to-1 pixel correspondence mode, that is, one monitor pixel displays the signal from a corresponding single sensor pixel, the simplest case scenario. In this display mode, only a portion of the image may be visible on the monitor, depending on the actual total number of pixels across the image, the final magnification, and the difference in the number of pixels between the camera sensor and monitor [11]. Figure 1 shows two examples of modern light microscopes: (a) a conventional stereo microscope with eyepieces, a digital camera, and two sizes of display monitor, and (b) a direct digital microscope with 2 sizes

\begin{tabular}{|c|c|c|c|c|c|}
\hline Image sensor megapixels & Pixels $^{a}$ & 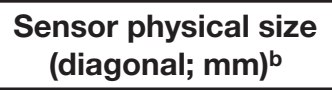 & $\begin{array}{c}\text { Sensor width } \\
(\mathrm{mm})\end{array}$ & $\begin{array}{l}\text { Sensor height } \\
\text { (mm) }\end{array}$ & $\begin{array}{c}\text { Sensor pixel size } \\
(\mu \mathrm{m})\end{array}$ \\
\hline 2.5 & $1,824 \times 1,368$ & 7.64 & 6.1 & 4.6 & 3.34 \\
\hline 5.04 & $2,592 \times 1,944$ & 7.64 & 6.1 & 4.6 & 2.35 \\
\hline 9.98 & $3,648 \times 2,736$ & 7.91 & 6.44 & 4.6 & 1.67 \\
\hline 19.96 & $5,472 \times 3,648$ & 15.86 & 13.2 & 8.8 & 2.4 \\
\hline
\end{tabular}
of display monitor, but without eyepieces.

Table 1: Specifications of image sensors used in digital cameras and microscopes supplied by Leica Microsystems.

aBy convention the sensor width is given first.

bSensor sizes are sometimes still given in arcane terms that originated in the vacuum tube era, e.g., 1/2.3" means a sensor 6.1 × 4.6 mm. Even then a 1/2.3" sensor from different manufacturers may have slightly different dimensions. 
Table 2: Examples of high definition displays including both computer monitors and TVs.

\begin{tabular}{|c|c|c|c|c|c|}
\hline $\begin{array}{l}\text { HD Flat Display } \\
\text { (inches) }\end{array}$ & $\begin{array}{l}\text { Display width } \\
\text { (mm) }\end{array}$ & $\begin{array}{c}\text { Display height } \\
\text { (mm) }\end{array}$ & $\begin{array}{l}\text { Pixel Size } \\
\quad(\mathrm{mm})\end{array}$ & Pixels & Megapixels \\
\hline PC monitor 21.5" & 476 & 267 & 0.25 & $1,920 \times 1,080$ & 2.07 \\
\hline PC monitor 24" & 521 & 324 & 0.27 & $1,920 \times 1,200$ & 2.3 \\
\hline PC monitor 27" & 597 & 337 & 0.31 & \multirow{7}{*}{$1,920 \times 1,080$} & \multirow{7}{*}{2.07} \\
\hline TV $32 "$ & 699 & 394 & 0.36 & & \\
\hline TV 40" & 880 & 495 & 0.46 & & \\
\hline TV 48" & 1,054 & 593 & 0.55 & & \\
\hline TV 55" & 1,211 & 681 & 0.63 & & \\
\hline TV 65" & 1,429 & 804 & 0.74 & & \\
\hline TV 75" & 1,648 & 927 & 0.86 & & \\
\hline TV 79" & 1,734 & 976 & 0.45 & \multirow{3}{*}{$3,840 \times 2,160$} & \multirow{3}{*}{8.29} \\
\hline TV 84" & 1,860 & 1,046 & 0.48 & & \\
\hline TV 85" & 1,882 & 1,058 & 0.49 & & \\
\hline
\end{tabular}

Resolution. For optical instruments in general, resolution or resolving power is the ability to see fine details in an image. More specifically, resolving power is the ability to distinguish in an image adjacent points or lines of the object that are closely spaced. Sometimes the terms magnification and resolution are used synonymously, but this is incorrect. Only resolution determines the limit of the smallest object that can be resolved. Magnification denotes the size of the resolved object. In light microscopy, resolution is typically expressed in line pairs per millimeter observed when lines of various separations are used as the object. In other words, at a given level of resolution pairs of black and white lines with equal line thickness and spacing can be distinguished. High magnification without sufficient resolution leads to empty magnification, as mentioned above [10]. Therefore, it is of vital importance to understand the limiting factors for resolution, not just for digital light microscopy, but for all forms of microscopy.

\section{Image Sensor and Display Monitor Combinations}

Pixel number and size. Image sensors used in most microscope digital cameras have a number of pixels typically between $1,600 \times 1,200$ and $5,472 \times 3,648$ and a pixel size between $1.5 \mu \mathrm{m}$ and $6 \mu \mathrm{m}$ (see examples in Table 1 ). High-definition (HD) electronic displays, including computer monitors and televisions, typically have minimum pixel numbers of $1,200,1,080$, or 2,160 pixels and pixel sizes between 0.12 and $0.9 \mathrm{~mm}$ (see Table 2) [12, 13]. Therefore, monitor pixels are typically 20 to 600 times larger than camera pixels.

Pixel size ratio. Knowing pixel sizes of image sensors (Table 1) and flat-screen HD monitors (Table 2), values for pixel size ratios can be calculated using Equation 5 (see Table 3).

\section{Digital Microscopes and Stereo Microscopes with Digital Cameras}

Total magnification. For simplicity, only two examples of digital microscopy are described here: a digital microscope and a stereo microscope equipped with a digital camera installed with a C-mount. It is assumed that in each case an image is displayed, using a 1-to-1 camera-to-monitor pixel correspondence, onto an HD monitor where sizes range from 21.5" (diagonal dimension 21.5 inches [54.6 cm]) to 75" (diagonal dimension 74.5 inches $[189 \mathrm{~cm}]$ ). Both microscopes use a $5 \mathrm{MP}$ image sensor. Table 4 shows examples of total magnification values (expressed as image size divided by object size) obtainable with the digital microscope or stereo microscope with digital camera.

For the digital microscope, the magnification range for the objective lens is $0.32 \times$ to $2 \times$, and the tube factor (q) including the photographic projection lens has a maximum to minimum magnification range of $8: 1$ (ratio of max to min tube factor magnification). For the stereo microscope with camera, the magnification range for the objective is $0.5 \times$ to $2 \times$, for the zoom from $0.78 \times$ to $16 \times$, for the eyepieces $10 \times$ to $25 \times$, and for the C-mount lens from $0.4 \times$ to $1 \times$. Using Equations 2 and $4 \mathrm{~b}$, example calculations for the minimum and maximum magnification values $\left(M_{T O T}\right.$ VIS and $M_{D I S}$ in table 4) achievable with the stereo microscope having a $5 \mathrm{MP}$ camera sensor can be shown. The stereo microscope has a min and max objective magnification of $M_{O}=0.5 \times$ or $2 \times$, a min and max zoom factor of $q=0.78 \times$ or 16×, a min and max C-mount magnification of $M_{P H O T}=$ $0.4 \times$ or $1 \times$, and a min and max eyepiece magnification of $M_{E}=10 \times$ or $25 \times$. Then the minimum and maximum total magnification $\left(M_{\text {TOT VIS }}\right)$ seen via the eyepieces would be:

Minimum: $\quad M_{\text {TOT VIS }}=0.5 \times \cdot 0.78 \times \cdot 10 \times=3.9 \times$

Maximum: $\quad M_{\text {TOT VIS }}=2 \times \cdot 16 \times \cdot 25 \times=800 \times$.

Also, the total display magnification $\left(M_{D I S}\right)$ seen via an image displayed on a 21.5 " or 75 " monitor (table 2) with a pixel size ratio of 106:1 and 366:1 (table 3 ) would be:

Minimum: $\quad M_{D I S}=0.5 \times \cdot 0.78 \times \cdot 0.4 \times \cdot 106=16.5 \times$.

Maximum: $\quad M_{D I S}=2 \times \cdot 16 \times \cdot 1 \times \cdot 366 \approx 11,700 \times$.

Magnifications on large monitors. Question: Which monitor size and pixel size would be needed to attain a total lateral display magnification of 30,000:1? An example can be shown using the stereo microscope with 5 MP camera mentioned above and Equations $3 b, 4 b$, and 5 . The maximum 
magnification from a stereo microscope for an image of the sample projected onto the camera sensor is:

max magnification onto sensor $=2 \times($ objective $) \cdot 16 \times($ zoom $) \cdot$ $1 \times($ C-mount $)=32 \times$.

The pixel ratio value corresponding to a total magnification of $30,000: 1$ with the above magnification of $32 \times$ onto the sensor is:

$$
\text { pixel ratio }=\frac{30,000(\text { total magnification })}{32(\text { sample to sensor magnification })}=938: 1
$$

The pixel size of the camera's $5 \mathrm{MP}$ sensor is $2.35 \mu \mathrm{m}$. Using the pixel ratio value of 938:1 and a 1-to-1 camera-to-monitor pixel correspondence, the monitor pixel size must be:

$$
\text { monitor pixel size }=938 \cdot 0.00235 \mathrm{~mm}=2.2 \mathrm{~mm}
$$

Therefore, to achieve a total magnification of 30,000:1 with a $5 \mathrm{MP}$ image sensor containing 2,592 horizontal pixels, the monitor horizontal size (assuming 1,920 pixels (HD) of $2.2 \mathrm{~mm}$ ) would be 4.2 meters (13.8 feet)! This width is more than 2.2 times larger than that of the largest monitor $(1.882 \mathrm{~m}$, UHD/4k) in Table 2.

But that is not the whole story. The distance the viewer is from a large monitor is also important for assessing what the viewer will see. If the viewer is too close, it is likely that the large pixels will be annoyingly visible. On the other hand, if the viewer is too far away, the finer details may be too small to see.

\section{Range of Useful Magnification for Digital Microscopy}

Now one must ask the question if this level of magnification, 30,000:1, is simply beyond the useful range, meaning it is empty magnification. How do we determine a range of useful magnification for digital microscopy when an image is observed on a monitor? First, it is important to understand the resolution or resolving power of the microscope system and the viewing distance from the monitor.

Microscope system resolution. The system resolution for a digital microscope (or stereo microscope with a digital camera) is influenced by three main factors:

(1) Diffraction-limited light microscope resolution, using Rayleigh's criterion and the numerical aperture [9]:

light resolution limit $=\frac{N A \cdot 10^{6}}{0.61 \cdot \lambda} \quad($ line pairs $/ \mathrm{mm})$

Table 3: Pixel size ratios (Equation 5) for HD monitors (Table 2) and image sensors used in digital microscopes and cameras supplied by Leica Microsystems (Table 1).

\begin{tabular}{|c|c|c|c|c|c|c|c|c|c|}
\hline \multirow{2}{*}{\begin{tabular}{c} 
Image $\begin{array}{c}\text { sensor } \\
\text { type }\end{array}$ \\
\cline { 2 - 10 }
\end{tabular}} & \multicolumn{8}{|c|}{ Monitor Size (inch) } \\
\cline { 2 - 9 }$y$ & \multicolumn{8}{|c|}{ Pixel Ratio } \\
\hline $2.5 \mathrm{MP}$ & $147: 1$ & $135: 1$ & $258: 1$ & $222: 1$ & $165: 1$ & $108: 1$ & $93: 1$ & $81: 1$ & $75: 1$ \\
\hline $5.04 \mathrm{MP}$ & $209: 1$ & $192: 1$ & $366: 1$ & $315: 1$ & $234: 1$ & $153: 1$ & $132: 1$ & $115: 1$ & $106: 1$ \\
\hline $9.98 \mathrm{MP}$ & $293: 1$ & $270: 1$ & $515: 1$ & $443: 1$ & $329: 1$ & $216: 1$ & $186: 1$ & $162: 1$ & $150: 1$ \\
\hline $19.96 \mathrm{MP}$ & $204: 1$ & $188: 1$ & $358: 1$ & $308: 1$ & $229: 1$ & $150: 1$ & $129: 1$ & $113: 1$ & $104: 1$ \\
\hline
\end{tabular}

where NA is the numerical aperture and $\lambda$ is the wavelength of light in $\mathrm{nm}$.

(2) Image sensor (camera sensor) resolution [9]:

$$
\begin{aligned}
& \text { sensor resolution limit }= \\
& \left.\frac{500 \cdot M_{\text {TOT PROJ }}}{\text { sensor bin mode } \cdot \text { sensor pixel size }} \text { (line pairs } / \mathrm{mm}\right)
\end{aligned}
$$

where $M_{\text {TOT PROJ }}$ is the magnification from the sample to the sensor (Equation 3), the "sensor bin mode" refers to the binning mode which is 1 for full frame $(1 \times 1)$ and 2 for $2 \times 2$ pixel binning, (see Figure 2a), and "pixel size" refers to the sensor pixel size in $\mu \mathrm{m}$.

(3) Display (monitor) resolution [9]:

monitor resolution limit $=$

$$
\left.\frac{M_{D I S}}{2 \cdot \text { monitor pixel size }} \quad \text { (line pairs } / \mathrm{mm}\right)
$$

where $M_{D I S}$ is the total lateral magnification (Equation 4) and the monitor pixel size is in $\mathrm{mm}$.

The basis for the camera sensor and display monitor resolution limit is the Nyquist sampling theorem for digital signal processing (see Figure 2b) $[14,15]$. This theorem assumes that at least two pixels are needed to resolve one line pair. One can simply imagine a microscope image of a sample showing line pairs projected onto the camera sensor and then displayed on the monitor. If a single line in the image corresponds to a single line of pixels, as shown in Figure $2 \mathrm{~b}$, say for the monitor, then to determine the minimum line pair spacing on the sample resolvable by the monitor, one can just divide the size of 2 pixels by the total lateral display magnification $\left(M_{D I S}\right)$. If the same exercise is done now for the camera sensor, that is, a single line in the image corresponds to a single line of pixels, then dividing by the total projection magnification from sample to sensor $\left(M_{\text {TOT PROJ }}\right)$ determines the minimum line pair spacing resolvable by the camera. To write these calculations out for more clarity:

Monitor: smallest resolvable line pair spacing sample $=$

$$
\frac{2 \cdot \text { monitor pixel size }}{M_{D I S}} \text {. }
$$

Camera sensor: smallest resolvable line pair spacing sample $=$

$$
\frac{2 \cdot \text { camera pixel size }}{M_{\text {TOT PROJ }}} \text {. }
$$

The reciprocal of the minimum resolvable line pair spacing gives the resolution limits shown above in Equations 7 and 8 . Simply add a conversion ( $\mu \mathrm{m}$ to $\mathrm{mm}$ ) and pixel binning factor for the camera sensor to arrive at the final form of Equation 7.

For this article, as stated above, the scenario of a 1-to-1 correspondence is assumed between the pixels of the sensor and monitor. For this specific case, using Equation $4 \mathrm{~b}$ and converting the monitor pixel size units from $\mathrm{mm}$ to $\mu \mathrm{m}$, it becomes clear that the resolution limit of the sensor and monitor are identical. Example calculations demonstrating this will be given in a later section of this article. 


\section{Monarc}

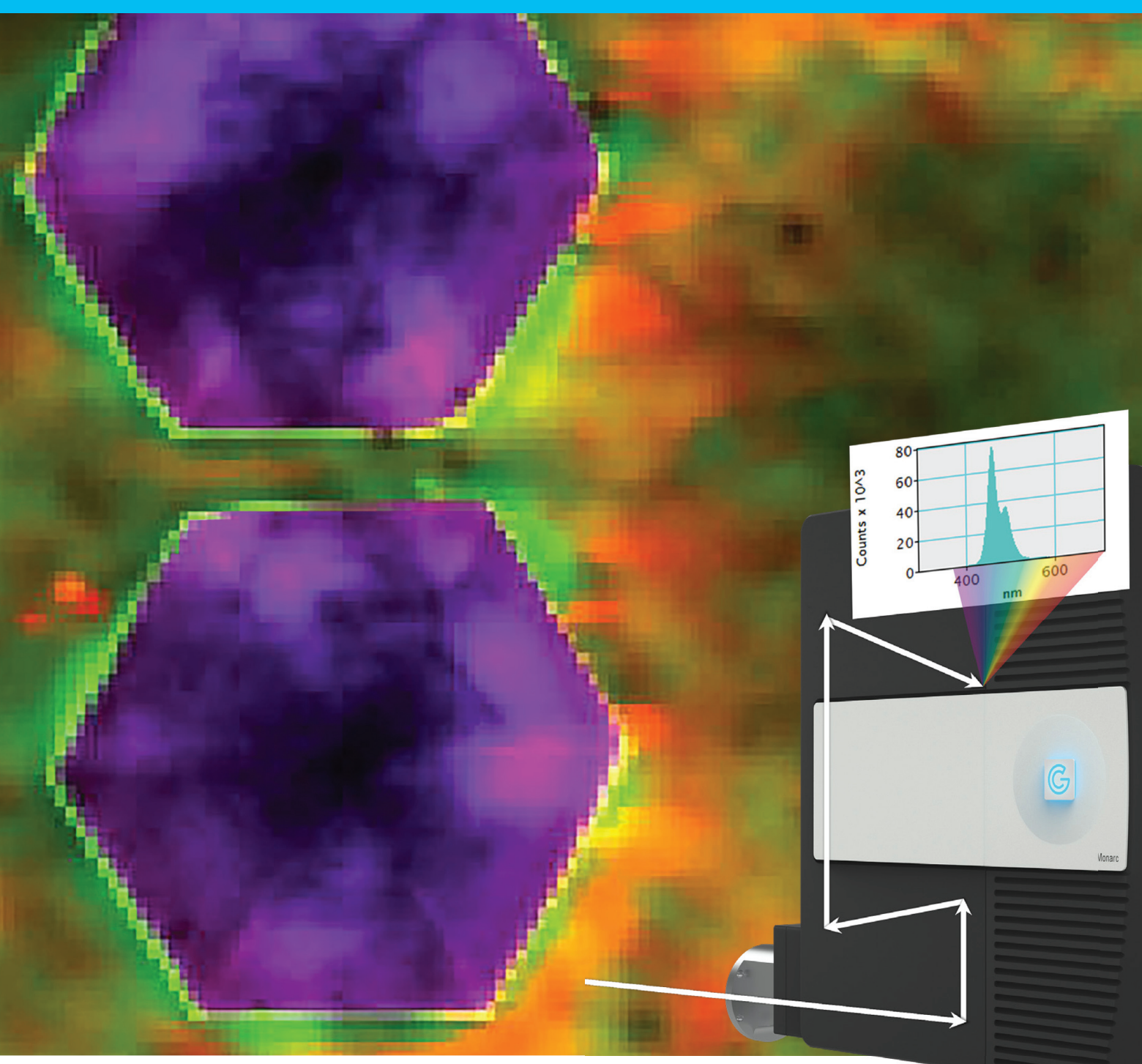

Introducing the Monarc Cathodoluminescence System Redefining what's possible in CL microscopy

The Monarc ${ }^{\mathrm{M}}$ offers the most powerful analysis of optical properties below the diffraction limit. Revealing the wavelength, angle, and polarization distributions of luminescence correlated directly with sample structure and composition at spatial resolutions better than $10 \mathrm{~nm}$.

Image: Composite wavelength-filtered image extracted from a hyperspectral data cube acquired with the Monarc of a GaN/InGaN MOW sample with V-pit defects (R=504 $\pm 2 \mathrm{~nm}, \mathrm{G}=435 \pm 2 \mathrm{~nm}, \mathrm{~V}=365 \pm 2 \mathrm{~nm}$ ). 
Table 4: Total magnification data, $\mathrm{M}_{\text {TOT }}$ VIS and $\mathrm{M}_{\mathrm{DIS}}$ (Equations 2 and 4b), for a digital microscope with $5 \mathrm{MP}$ image sensor (DMS1000) and a stereo microscope (M205 A) equipped with a 5 MP digital camera. The possible range of magnification values, minimum to maximum, for the discussed HD monitor sizes (Table 2) and pixel ratios (Table 3) are shown.

\begin{tabular}{|c|c|c|c|c|c|c|c|}
\hline \multicolumn{3}{|c|}{ Digital microscope with 5 MP sensor } & \multicolumn{5}{|c|}{ Stereo microscope with 5 MP camera } \\
\hline \multicolumn{2}{|c|}{ Monitor Size (inch) } & & \multicolumn{2}{|c|}{ Eyepiece } & \multicolumn{2}{|c|}{ Monitor Size (inch) } & \\
\hline $21.5 ”$ & $75 ”$ & & $10 x$ & $25 x$ & $21.5 ”$ & $75 ”$ & \\
\hline \multicolumn{2}{|c|}{$M_{D I S}$} & & \multicolumn{2}{|c|}{ MTOT VIS } & \multicolumn{2}{|c|}{$M_{D I S}$} & \\
\hline $8.4 \times$ & $29 \times$ & $\min$ & $3.9 x$ & $9.75 \times$ & $16.5 \times$ & $57 \times$ & $\min$ \\
\hline $420 x$ & $1,450 \times$ & $\max$ & $320 x$ & $800 x$ & $3,400 \times$ & $11,700 x$ & $\max$ \\
\hline
\end{tabular}

The resolution limit of the digital microscope system resolution is determined by the smallest of the three resolution values above. The diffraction limit of the light microscope (Equation 6) still governs the ultimate level of detail that can be observed and recorded. This last point is important: the best resolution of a microscope generally is measured from a recorded image rather than a viewed image; methods for this fall outside the topics of this article [16].

Useful viewing distance. The viewing distance is the distance between the observer's eyes and the displayed image. The range for a useful viewing distance is affected by the system resolution of the microscope and the visual angle of the observer $[17,18]$. The minimal angle of resolution depends on the intensity of light emitted or reflected from the observed object and the contrast between its specific features. The angle ranges, on average, from 2.3 to 4.6 minutes of arc (low to high light intensity and contrast) for human eyes $[9,19-21]$. This angular range indicates the optimal performance, in terms of visual acuity (resolution) and contrast sensitivity, of the human eye averaged over a large population varying in age from young to old. Thus, on average, an eye is capable of distinguishing details on a monitor which have a separation distance corresponding to an angular difference of 2.3 to 4.6 minutes of arc (0.038 to 0.077 degrees; 0.669 to $1.338 \times$ $10^{-3}$ radians) for a specific viewing distance. To understand how the range for a useful viewing distance is determined, imagine a person observing an image displayed on a monitor which shows the smallest line pair spacing resolvable by the digital microscope system. It follows that the actual line pair spacing on the sample would then be:

minimal line pair spacing observed monitor $=$

$\mathrm{M}_{\text {DIS }} \cdot$ (minimal resolvable line pair spacing sample)

where $M_{D I S}$ is the total magnification (Equation 4). To determine the minimal angle of resolution for the observer's eye necessary in order to see two separate lines in the pair, the minimal line spacing observed on the monitor must be divided by the viewing distance:

min angle resolution for observer $=$

\section{$\frac{M_{D I S} \cdot(\text { min resolvable line pair spacing sample) }}{\text { viewing distance }}$ (radians).}

The minimal resolvable line pair spacing on the sample is the inverse of the microscope system resolving power or resolution, thus:

min angle resolution observer $=$

$$
\frac{M_{D I S}}{\text { system resolution } \cdot \text { viewing distance }}
$$

(radians).
As noted above, the minimum angle of resolution for the eye falls between $6.669 \times 10^{-4}$ and $1.338 \times 10^{-3}$ radians, so rearranging the equation above for viewing distance (in $\mathrm{mm}$ ) and setting it equal to the lower and upper values of the angle of resolution, the useful viewing distance range can be expressed as (converted from units of $\mathrm{mm}$ to meters):

$$
\begin{aligned}
0.75 \cdot \frac{M_{D I S}}{\text { system resolution }}<\text { useful viewing distance }< \\
1.5 \cdot \frac{M_{\text {DIS }}}{\text { system resolution }} \text { (meters) }
\end{aligned}
$$

Again, $M_{D I S}$ is the total lateral magnification (Equation 4), and the system resolution refers to the light microscope system resolution limit as discussed above (Equations 6-8). For the following calculations, it is assumed that the viewing distance is always within the useful range.

Range of useful magnification. To understand how to determine the range of useful magnification for digital microscopy - the lowest and highest magnification values for an image displayed on a monitor where the system resolution limit is clearly observed for optimal and non-optimal illumination-it is first necessary to mention briefly the "perceived" magnification from visual observation of an image or object. Everyday experience shows that the perceived size of an object depends on the distance from which it is observed. Using geometrical optics and the relationship between angular and lateral magnification, the following can be derived:

$$
\text { visual magnification }=\frac{M_{D I S} \cdot(250 \mathrm{~mm})}{\text { viewing distance }} \text { (no units) }
$$

where $M_{D I S}$ is the total magnification (Equation 1) and 250 refers to the standard reference for the viewing distance in $\mathrm{mm}$, which is based on the average near point for the human eye, that is, the closest point on which the eye can focus [22]. The Appendix at the end of this article provides a more detailed derivation of Equation 10.

Finally, the range of useful magnification can be defined by combining Equations 9 and 10. From Equation 10, after converting the near point of the eye from units of $\mathrm{mm}$ to meters:

$$
\text { viewing distance }=\frac{(0.25 \mathrm{~m}) \cdot M_{D I S}}{\text { visual magnification }}
$$

Then, substituting the expression just above for the viewing distance into Equation 9 and dividing all sides by 0.25 and $M_{\text {DIS }}$ : $\frac{0.75}{0.25 \cdot \text { system resolution }}<\frac{1}{\text { useful magnification }}<$ 

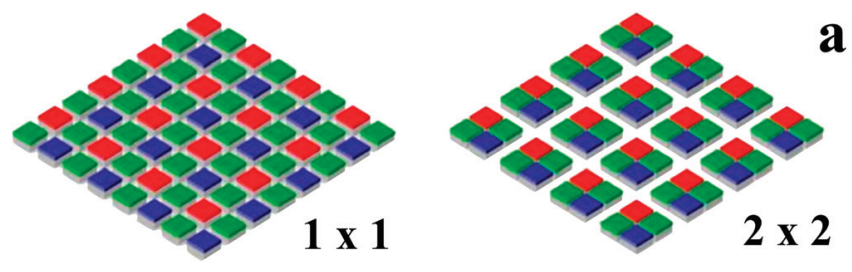

a
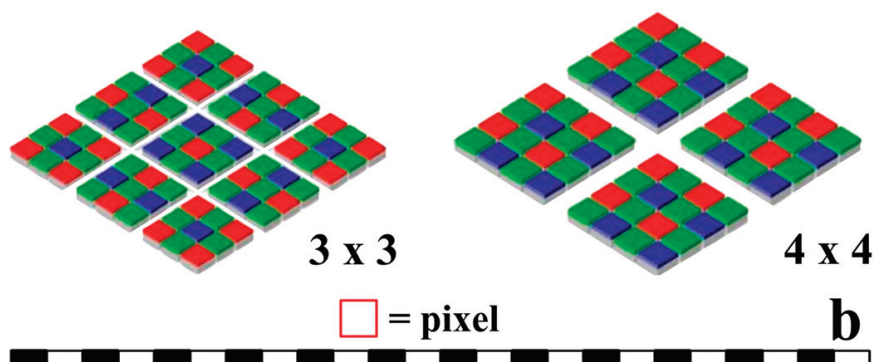

b

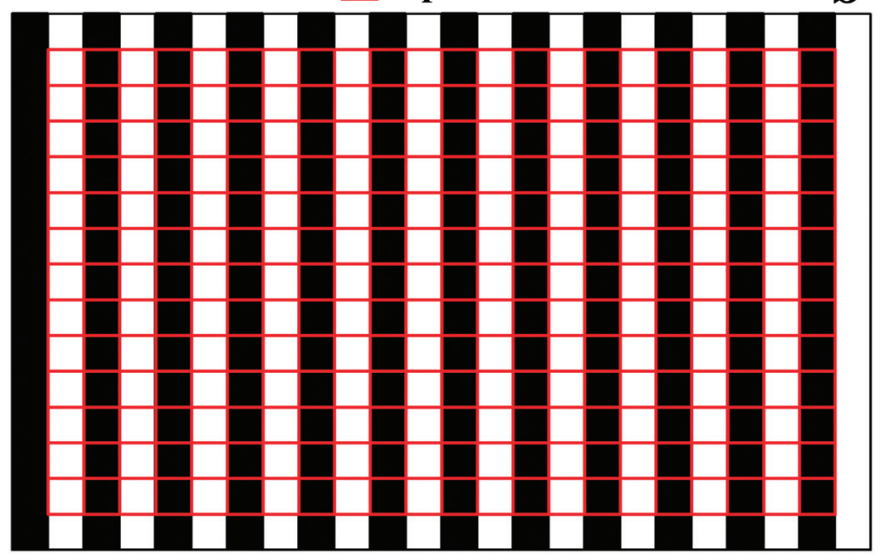

Figure 2: (a) Examples of pixel binning modes for image sensors: no binning (full frame, $1 \times 1)$, double binning $(2 \times 2)$, triple binning $(3 \times 3)$, and quadruple binning $(4 \times 4)$. (b) Image sensor detection of black/white line pairs, used to measure the resolution limit of a microscope, requires a minimum of two pixels (red squares) per line pair (Nyquist rate). However, better image results are obtained if three or more pixels per line pair are used.

Now, by taking the reciprocal of the inequality, remembering to interchange the greater than and lesser than signs, the range of useful magnification is determined:

$$
\begin{gathered}
\frac{\text { system resolution }}{6}<\text { useful magnification }< \\
\frac{\text { system resolution }}{3} \text { (no units) }
\end{gathered}
$$

Thus, the range of useful magnification is between $1 / 6$ and $1 / 3$ of the microscope system resolution.

What does it mean exactly, the range of useful magnification? It means the optimal magnification range when observing an object via a microscope (or an optical instrument that can magnify) where the finest resolvable features can be seen. Remember that range is based on the average performance of the human eye with respect to visual acuity (resolution) and contrast sensitivity along with the optical system's resolution limit, as mentioned above. A significant number of individuals will have eyes who perform above or below this average.

Low magnification. When the magnification from sample to camera sensor is low, generally $1 \times$ or even less, then the camera sensor or monitor are the limiting resolution factors of the microscope system. As an example, a digital microscope using a $1.6 \times$ objective $\left(M_{O}=1.6 \times\right)$ with a numerical aperture of $0.05(N A=0.05)$, a total tube factor of $1 \times(q=1 \times)$, a $0.32 \times$ photographic projection lens $\left(M_{P H O T}=0.32 \times\right)$, a camera sensor with a $3 \mu \mathrm{m}$ pixel size and binning mode of $1 \times 1$, a monitor pixel size of $0.3 \mathrm{~mm}$ (pixel ratio of 100:1), a 1-to-1 pixel correspondence between the sensor and monitor, and white light (mixture of all visible light wavelengths [400-700 nm] with an average in the green at $\lambda=550 \mathrm{~nm}$ ) illumination, then from Equations 6, 7, and 8:

$$
\text { light resolution limit }=\frac{0.05 \cdot 10^{6}}{0.61 \cdot 550}=149 \text { line pairs } / \mathrm{mm}
$$

sensor resolution limit $=\frac{500 \cdot 1.6 \cdot 1 \cdot 0.32}{1 \cdot 3}=85$ line pairs $/ \mathrm{mm}$; and monitor resolution limit $=\frac{1.6 \cdot 1 \cdot 0.32 \cdot 100}{2 \cdot 0.3}=85$ line pairs $/ \mathrm{mm}$.

This example shows that, at such low magnification, the resolution limit of camera sensors with pixels sizes larger than $2 \mu \mathrm{m}$ (and monitors with pixel sizes larger than $0.2 \mathrm{~mm}$ ) will start to be inferior to the light resolution. Therefore, at low magnification, approximately $1 \times$ or less, the sensor or monitor will likely be the limiting factor for the microscope system resolution.

High magnification. When the magnification from sample to camera sensor is high, generally $50 \times$ or greater, then light diffraction is the limiting resolution factor of the microscope system. Again, to illustrate it with an example, a digital microscope using a $160 \times$ objective $\left(M_{O}=160 \times\right)$ with a numerical aperture of $1.4(N A=1.4)$, a total tube factor of one $(q=1 \times)$, a $1 \times$ photographic projection lens $\left(M_{P H O T}=1 \times\right)$, a camera sensor with a $6 \mu \mathrm{m}$ pixel size and binning mode of $1 \times 1$, a monitor pixel size of $0.3 \mathrm{~mm}$ (pixel ratio of 50:1), a 1-to-1 pixel correspondence between the sensor and monitor, and green light $(\lambda=550 \mathrm{~nm})$ illumination, then from Equations 6, 7, and 8:

$$
\text { light resolution limit }=\frac{1.4 \cdot 10^{6}}{0.61 \cdot 550}=4,173 \text { line pairs } / \mathrm{mm} \text {; }
$$

sensor resolution limit $=\frac{500 \cdot 160 \cdot 1 \cdot 1}{1 \cdot 6}=13,333$ line pairs $/ \mathrm{mm}$; and monitor resolution limit $=\frac{160 \cdot 1 \cdot 50}{2 \cdot 0.3}=13,333$ line pairs $/ \mathrm{mm}$.

Here at high sample-to-sensor magnification, the system resolution of a microscope using a modern camera sensor with a pixel size in the $1-6 \mu \mathrm{m}$ range (and a monitor pixels size below $0.6 \mathrm{~mm}$ ) is limited by the light resolution.

For a best-case scenario, the greatest light resolution possible with the smallest wavelength of visible light, $400 \mathrm{~nm}$, and a very high numerical aperture, 1.4, is approximately 5,740 line pairs $/ \mathrm{mm}$. From the example above, it is clearly seen that the resolution limit of a camera sensor with a pixel size below $6 \mu \mathrm{m}$ easily exceeds this value.

For this description of digital microscopy, it is assumed that the image on the monitor is always observed within the 

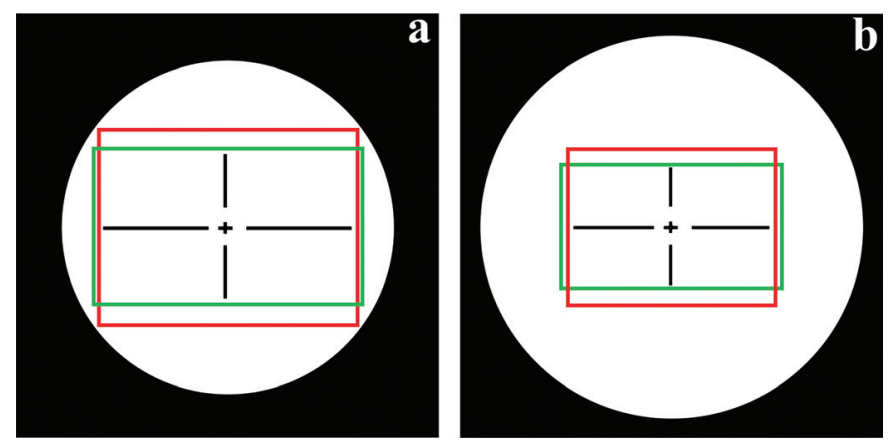

Figure 3: Diagram showing direct comparison of an image viewed through eyepieces (white circle) and simultaneously with the sensor (rectangles) of a 5 MP digital camera. The two examples shown are: (a) eyepiece with a field number (FN) of $20 \mathrm{~mm}$ and C-mount with $0.4 \times$ lens, and (b) eyepiece with $23 \mathrm{~mm} F \mathrm{FN}$ and C-mount with $0.5 \times$ lens. Some cameras detect images in a $4: 3$ aspect ratio (red rectangle) format for data storage and a 16:9 aspect ratio (green rectangle) format for live image output.

range of useful viewing distance. As discussed above, the range of useful magnification is derived based on the average performance of the eye. Whenever a sample is observed with a light microscope, sample features that correspond to the microscope's resolution limit become resolvable by the eye under optimal illumination conditions at the lower magnification value of the useful range (Equation 11). For non-optimal illumination, then the higher magnification value may be necessary for the eye to resolve the features. Beyond the higher magnification value of the range, no finer details of the sample can be resolved, so it is empty magnification.

The example above for high magnification demonstrated that the best possible resolution attained with a light microscope (objective with a 1.4 numerical aperture and illumination with $400 \mathrm{~nm}$ light) is about 5,740 line pairs/ $\mathrm{mm}$. Now, the range of useful magnification for the best resolution case can be calculated using Equation 11:

$$
\begin{gathered}
\frac{5,740}{6}<\text { useful magnification }<\frac{5,740}{3} \\
957<\text { useful magnification }<1,913 .
\end{gathered}
$$

So the highest useful magnification for a light microscope is about $1,900 \times-2,000 \times$. Magnification values exceeding $2,000 \times$ fall under empty magnification. When below the lower end of the range, 957 $\times$, again it means that the average eye can no longer distinguish details on a sample with a spatial frequency equivalent to 5,740 line pairs $/ \mathrm{mm}$. However, most samples are not uniform with features of the same exact dimensions and spatial frequency everywhere on the surface. Normally, as magnification is decreased, other larger features with lower spatial frequencies (below the resolution limit) become resolvable.

The example for low magnification above, where the image sensor limits the resolution of the microscope (objective with a 0.05 numerical aperture and sensor with $3 \mu \mathrm{m}$ pixel size), shows a limit of about 85 line pairs $/ \mathrm{mm}$. Now, the range of useful magnification for this low magnification case also can be calculated:

$$
\begin{aligned}
& \frac{85}{6} x<\text { useful magnification }<\frac{85}{3} \times \\
& 14 x<\text { useful magnification }<28 \times .
\end{aligned}
$$

So the range of useful magnification for this case, where the sensor limits the resolution of the microscope, is about $14 \times-28 \times$. In order to resolve features on a sample with a spatial frequency equivalent to 85 line pairs $/ \mathrm{mm}$, the total magnification $\left(M_{D I S}\right)$ of the observed image should fall between $14 \times$ and $28 \times$. Beyond $28 \times$ it would be empty magnification. To make sure that sample features with a spatial frequency corresponding to the resolution limit of 85 line pairs $/ \mathrm{mm}$ can be resolved, then the choice of a monitor with appropriate dimensions and pixel size becomes important.

\section{Object Field (Field of View)}

An object field $(\mathrm{OF})$ is the part of the object that is reproduced in the final image. It is also known as the microscope field of view (FOV). Thus, details of an object can only be observed if they are present within the OF. When looking through the eyepieces, the visible $\mathrm{OF}$ is a circular image of a portion of the sample. The size of the OF (Equation 12) is dependent on the field number (FN) of the eyepiece, as well as the magnification of the objective and tube lenses (Figure 3). In digital microscopy, the OF is rectangular because of the shape of the image sensor that collects the image and the monitor that displays it (see Figure 3). It is expressed in width and height given in $\mathrm{mm}$. For digital microscopy, care has to be taken that the image created by the optical system is large enough to cover the whole image sensor. The OF can be limited either by the image sensor or the display. In either case, the physical size of the active area, given by the number of active pixels in width and height and their physical size (pixel pitch), has to be taken into account.

To calculate the OF, the physical size of the active area of the sensor (Equation 13) must be divided by either the magnification of the objective, tube, and camera projection lenses $\left(M_{\text {TOT PROJ }}\right)$ or, for the monitor, by the total lateral display magnification, $M_{\text {DIS. The smaller of these values for }}$ each width and height define the digital microscope's OF. It is likely that both width and height of the OF are not necessarily jointly limited by the image sensor or display. For example, width can be limited by the sensor, whereas the height can be limited by the display. The final OF will depend on the dimensions and aspect ratios of the image sensor and display and the pixel correspondence $(1: 1,1: 2$, $2: 1$, etc.) between them for image display. In this article, a 1-to-1 sensor pixel to monitor pixel correspondence is assumed (refer to section with Equation 5 above).

Eyepiece object field. The OF for eyepieces can be determined by:

OF $_{\text {eyepiece }}=\frac{F N}{M_{O} \cdot q}$

$(\mathrm{mm})$

where $O F_{\text {eyepiece }}$ is the object field observed through an eyepiece, $F N$ is the eyepiece field number in $\mathrm{mm}$, and $M_{O} \cdot q$ (Equation 2) is the total magnification before the eyepiece due to the objective, zoom, and any tube lenses. 
Sensor object field. The OF for a camera sensor can be determined using the width and height of the sensor divided by the total magnification of the optics producing the image of the sample projected onto the sensor:

$w=\frac{\text { pixel size } \cdot \text { number of active pixels in sensor width }}{1000 \cdot M_{\text {TOT РRОJ }}}(\mathrm{mm})$

$h=\frac{\text { pixel size } \cdot \text { number of active pixels in sensor height }}{1000 \cdot M_{\text {TOT РROI }}}(\mathrm{mm})$

where $w$ and $h$ are the width and height of the OF observed by

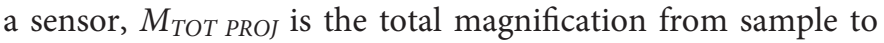
sensor (Equation $3 \mathrm{~b}$ ), and the sensor pixel size is in $\mu \mathrm{m}$.

Figures 3 and 4 show the difference in OF between images seen by the eyepieces versus those recorded by the camera sensor, for the same sample, objective lens, and zoom setting. For Figure 4, the total magnification of the objective and zoom lens was $1 \times$, but several types of C-mounts with different magnification were used to install the 5 MP camera on the stereo microscope. The red rectangle seen in Figure 4a represents the OF of Figure $4 b$, an image taken with the $0.32 \times$ $\mathrm{C}$-mount. The blue rectangle indicates the OF of Figure $4 \mathrm{c}$, taken with the $0.5 \times \mathrm{C}$-mount. The green rectangle shows the OF of Figure $4 d$, taken with the $0.63 \times \mathrm{C}$-mount. Figure $4 \mathrm{~b}$ shows the problem of vignetting where the edges of the image are darker than the center. Vignetting almost always occurs with stereo or compound microscopes having a $\mathrm{C}$-mount with too low a magnification. In that case, the image projected onto the camera sensor is smaller than the sensor size resulting in dark edges. To avoid such a problem, normally it is recommended that a $0.32 \times \mathrm{C}$-mount is used with a digital camera having a $4.8 \times 3.6 \mathrm{~mm}$ sensor size, a $0.4 \times$ C-mount with a $6.1 \times 4.6 \mathrm{~mm}$ sensor size, a $0.5 \times \mathrm{C}$-mount with a $8.0 \times 6.4 \mathrm{~mm}$ sensor size, and a $0.63 \times \mathrm{C}$-mount with a $8.8 \times 6.6 \mathrm{~mm}$ sensor size.

The OF of the camera sensor can be calculated $(w$ $\times h$ ) using Equation 13a and 13b above. The range of values for the OF seen with a digital microscope and stereo microscope equipped with a digital camera are shown in Table 5.

\section{Discussion}

In planning a set of experiments, it is useful to know the lowest magnification for which the object field does not exhibit vignetting, as well as the useful range of magnification to avoid introducing empty magnification. While these issues have been described for the cases of relatively low-magnification digital microscopes and stereo microscopes, typically used for inspection during manufacturing, the same concepts can be applied to other types of light microscopes, such as compound or higher-performance digital microscopes. Indeed, the issues of magnification range and magnification values related to various monitor sizes are quite general and they are relevant to other instruments, such as scanning electron microscopes, transmission electron microscopes, and scanning probe microscopes. While the concepts would remain the same, the equations for calculating specific values of magnification would need modification.

\section{Conclusion}

Digital microscopes use electronic image sensors (camera sensors) to replace eyepieces. Microscopes for direct visual perception, such as stereo and compound microscopes, have eyepieces and can be equipped with digital cameras. Digital microscopy allows rapid acquisition of high-quality images. It is often used for fast and easy documentation, quality control (QC), failure analysis, and research and development in a variety of fields. Because of the diversity of camera sensor dimensions and electronic display monitor sizes, determining magnification and magnification range when using digital microscopy can be challenging. With this article, users of digital microscopy can better understand how to evaluate the total magnification and its useful range for a particular microscope. The useful range of magnification is dependent upon the resolution of the optical instrument and average performance of the human eye with respect to visual acuity (angular resolution) and contrast sensitivity for optimal and non-optimal illumination conditions.

\section{Acknowledgments}

We would like to thank our colleagues Reto Züst and Harald Schnitzler of Leica Microsystems, Heerbrugg, Switzerland, for useful discussion and clarification about the many concepts and definitions concerning magnification and resolution described in the microscopy standards.
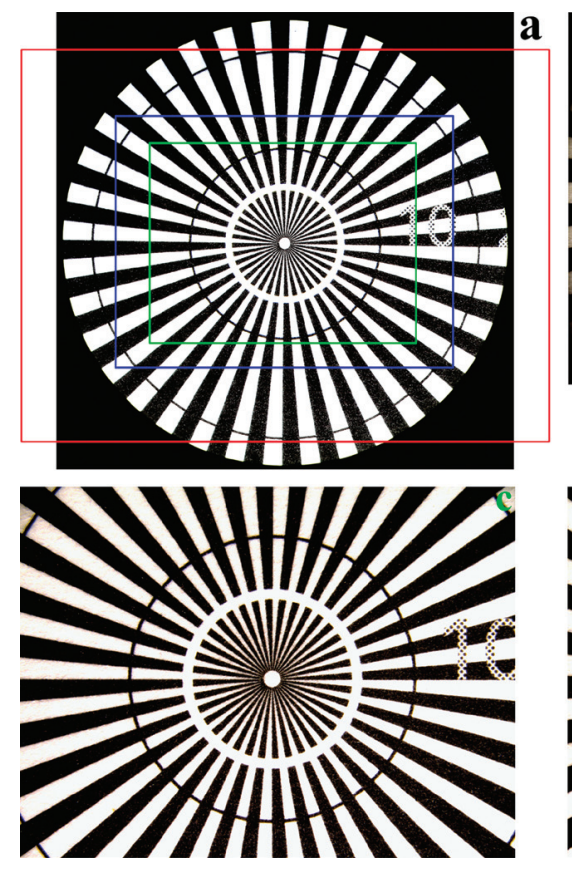

Figure 4: Images of a Siemens star taken with a stereo microscope (M205 A) having a tota objective and zoom lens magnification $\left(\mathrm{M}_{\mathrm{O}} \cdot \mathrm{q}\right)$ of $1 \times$. The first black line circle has a $10 \mathrm{~mm}$ diameter and the second a $20 \mathrm{~mm}$ diameter. (a) Image photographed through a 10x eyepiece with $23 \mathrm{~mm}$ field number (FN). Images (b-d) recorded using a digital camera installed with a C-mount (M $0.63 \times \mathrm{M}_{\text {PHOT }}$ and $13.8 \times 10.3 \mathrm{~mm}$ OF. The red rectangle in $4 \mathrm{a}$ represents the OF of $4 \mathrm{~b}(0.32 \times$ C-mount), the blue the OF of $4 \mathrm{c}(0.5 \times \mathrm{C}$-mount), and the green the OF of $4 \mathrm{~d}(0.63 \times \mathrm{C}$-mount). 
Table 5: Object field (OF) data (Equation 13) for an image from a digital microscope with 5 MP image sensor (DMS1000) and stereo microscope (M205 A) equipped with a 5 MP digital camera (MC170 HD) showing the range from minimum to maximum values. The digital and stereo microscope discussed in this article are used often for inspection, such as during manufacturing. Generally, their maximum magnification values are lower than compound or higher performance digital microscopes.

\begin{tabular}{|c|c|c|c|}
\hline $\begin{array}{l}\text { Digital microscope } \\
\text { with } 5 \text { MP sensor }\end{array}$ & \multicolumn{2}{|c|}{$\begin{array}{l}\text { Object field (OF) } \\
\text { of Sensor }\end{array}$} & \\
\hline MTOT PROJ & $\boldsymbol{w}(\mathrm{mm})$ & $\boldsymbol{h}(\mathrm{mm})$ & \\
\hline $0.08: 1(0.08 \times)$ & 77 & 58.1 & $\max \mathrm{OF}$ \\
\hline $3.96: 1$ (3.96x) & 1.5 & 1.2 & $\min O F$ \\
\hline $\begin{array}{l}\text { Stereo microscope } \\
\text { with } 5 \text { MP camera }\end{array}$ & \multicolumn{2}{|c|}{$\begin{array}{c}\text { Object field (OF) } \\
\text { of Sensor }\end{array}$} & \\
\hline MTOT PROJ & $\boldsymbol{w}(\mathrm{mm})$ & $\boldsymbol{h}(\mathrm{mm})$ & \\
\hline $0.16: 1(0.16 \times)$ & 39.1 & 29.5 & $\max O F$ \\
\hline $32: 1(32 x)$ & 0.19 & 0.14 & $\min O F$ \\
\hline
\end{tabular}

\section{References}

[1] C Hoyer, Chair Technical Committee, ISO 8039:2014: Microscopes -- Values, tolerances and symbols for magnification, International Organization for Standardization (ISO), Geneva, Switzerland, 2014, www.iso.org/standard/ 66260.html.

[2] C Hoyer, Chair Technical Committee, ISO 8578:2012:

Microscopes -- Marking of objectives and eyepieces, International Organization for Standardization (ISO), Geneva, Switzerland, 2012, www.iso.org/standard/50988. html.

[3] C Hoyer, Chair Technical Committee, ISO 9345-1:2012: Microscopes -- Imaging distances related to mechanical reference planes -- Part 1: Tube length $160 \mathrm{~mm}$, International Organization for Standardization (ISO), Geneva, Switzerland, 2012, www.iso.org/standard/61065.html.

[4] C Hoyer, Chair Technical Committee, ISO 9345-2:2014: Microscopes -- Imaging distances related to mechanical reference planes -- Part 2: Infinity corrected optical systems, International Organization for Standardization (ISO), Geneva, Switzerland, 2014, www.iso.org/standard/64986. html.

[5] C Hoyer, Chair Technical Committee, ISO 10934-1:2002: Optics and optical instruments -- Vocabulary for microscopy -- Part 1: Light microscopy, International Organization for Standardization (ISO), Geneva, Switzerland, 2002, www.iso.org/standard/35112.html.
[6] C Hoyer, Chair Technical Committee, ISO 10934-2: 2007: Optics and optical instruments -- Vocabulary for microscopy -- Part 2: Advanced techniques in light microscopy, International Organization for Standardization (ISO), Geneva, Switzerland, 2007, www.iso.org/standard/ 39473.html.

[7] C Hoyer, Chair Technical Committee, ISO 19012-1:2013: Microscopes -- Designation of microscope objectives -- Part 1: Flatness of field/Plan, International Organization for Standardization (ISO), Geneva, Switzerland, 2013, www.iso. org/standard/61652.html.

[8] C Hoyer, Chair Technical Committee, ISO 19012-2:2013: Microscopes -- Designation of microscope objectives -- Part 2: Chromatic correction, International Organization for Standardization (ISO), Geneva, Switzerland, 2013, www.iso. org/standard/61836.html.

[9] C Hoyer, Chair Technical Committee, ISO 18221:2016: Microscopes -- Microscopes with digital imaging displays -- Information provided to the user regarding imaging performance, International Organization for Standardization (ISO), Geneva, Switzerland, 2016, www. iso.org/standard/61810.html.

[10] A Schué, Beware of "Empty" Magnification, Science Lab, Leica Microsystems, Wetzlar, Germany, 2008, www.leica-microsystems.com/science-lab/ beware-of-empty-magnification.

[11] J DeRose and M Doppler, What Does 30,000:1 Magnification Really Mean? Some Useful Guidelines for Understanding Magnification in Today's New Digital Microscope Era, Science Lab, Leica Microsystems, Wetzlar, Germany, 2015, www.leica-microsystems.com/science-lab/ what-does-300001-magnification-really-mean.

[12] Computer display standard, Wikipedia The Free Encyclopedia, Wikipedia Foundation, San Francisco, 2015, en.wikipedia.org/wiki/Computer_display_standard.

[13] Display resolution, Wikipedia The Free Encyclopedia, Wikipedia Foundation, San Francisco, 2015, en.wikipedia.org/wiki/Display_resolution.

[14] CE Shannon, Proceedings IEEE 86(2) (1998) 447-57, ieeexplore.ieee.org/document/659497.

[15] H Nyquist, Proceedings IEEE 90(2) (2002) 280-305, ieeexplore.ieee.org/document/989875.

[16] S Bradbury, Basic Measurement Techniques for Light Microscopy, Royal Microscopy Society Handbook \#23, Oxford University Press, Oxford, UK, 1991, pp. 1-105.

[17] CG Healey and AP Sawant, ACM Trans Applied Perception 9(4) (2012); 20: 1-21.

[18] S Sugawara et al., SMPTE Motion Imag J 117(3) (2008) 23-29.

[19] T Kratzer, Chair Technical Committee, ISO 8596:2017: Ophthalmic optics -- Visual acuity testing -- Standard and clinical optotypes and their presentation, International 
Organization for Standardization (ISO), Geneva, Switzerland, 2017, www.iso.org/standard/69042.html.

[20] CD Hendley, J Gen Physiol 31(5) (1948) 433-57.

[21] H Strasburger et al., J Vision 11(5):13 (2011) 1-82.

[22] A Duane, Trans Am Ophthalmol Soc 20(1) (1922) 132-57.

\section{Appendix}

Derivation of Equation 10:

$$
\text { visual magnification }=\frac{M_{D I S} \cdot(250 \mathrm{~mm})}{\text { viewing distance }} \text { (no units). }
$$

The diagram in Fgure A1 shows the same object being observed by a person from two different distances. One distance is the so-called "viewing distance" $\left(d_{v d}\right)$ and the other is the standard reference for the viewing distance based on the average near point $\left(d_{n p}\right)$ of the human eye (the closest point on which the eye can focus), $250 \mathrm{~mm}$ [22]. The observed or perceived heights of the object are $h_{v d}$ at the viewing distance and $h_{n p}$ at the eye's near point. For rays of light coming from the top of the object and passing directly to the center of the eye's lens in a straight line, the rays subtend an angle with respect to the horizontal of $\alpha_{v d}$ for the object at the viewing distance position and $\alpha_{n p}$ at the eye's near point.

In Figure A2, an equivalent result as for Figure A1 is shown using an imaginary lens system that produces a virtual image of the object. The object is placed at the eye's near point $\left(d_{n p}\right)$, and the virtual image of the object appears at the viewing distance position $\left(d_{v d}\right)$. Now Figure A2 of the diagram can be analyzed using basic geometrical optics in terms of magnification. The angular magnification, $M_{A N G}$, and lateral magnification, $M_{D I S}$ (Equation 1), for Figure A2 are:

$$
M_{A N G}=\frac{\alpha_{v d}}{\alpha_{n p}} \quad \text { and } \quad M_{D I S}=\frac{h_{v d}}{h_{n p}} .
$$

Considering only the case of paraxial rays, then the angle $\alpha \approx \tan \alpha$. It can also be seen from Figure A2 that:

$$
\tan \left(\alpha_{v d}\right)=\frac{h_{v d}}{d_{v d}} \cong \alpha_{v d} \quad \text { and } \quad \tan \left(\alpha_{n p}\right)=\frac{h_{n p}}{d_{n p}} \cong \alpha_{n p} .
$$

Therefore,

$$
M_{A N G}=\frac{h_{v d} / d_{v d}}{h_{n p} / d_{n p}}=\frac{h_{v d} \cdot d_{n p}}{h_{n p} \cdot d_{v d}}=M_{D I S} \cdot \frac{d_{n p}}{d_{v d}} .
$$

Equating $M_{A N G}$ with the visual magnification and knowing that $d_{n p}$ is $250 \mathrm{~mm}$ and $d_{v d}$ the viewing distance, then:

$$
\text { visual magnification }=M_{\text {DIS }} \cdot \frac{250}{\text { viewing distance }}
$$

which is Equation 10.
For many cases, the viewing distance will be approximately the eye's near point $\left(d_{v d} \approx d_{n p}\right)$, so:

$$
\text { visual magnification } \approx M_{D I S}
$$
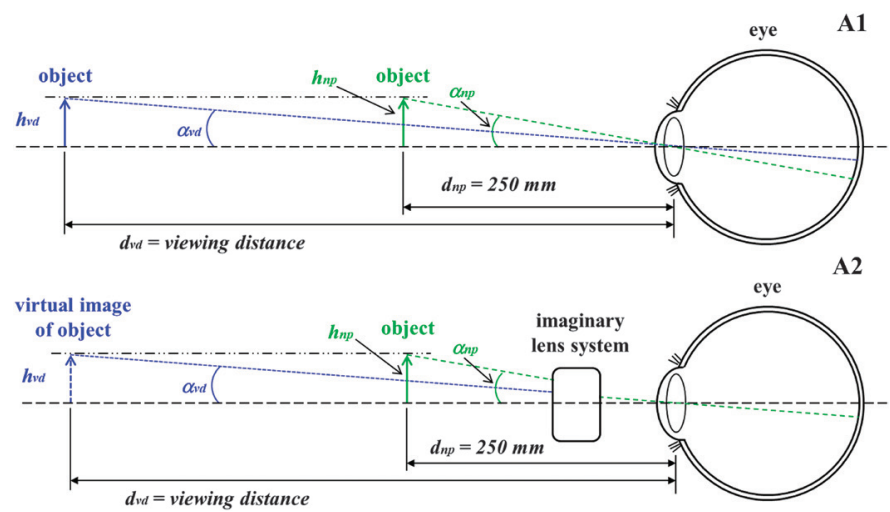

Figure A: (1) Diagram showing the same object observed by the unaided eye from two different distances, the "viewing distance" and average near point of the eye $(250 \mathrm{~mm})$. The viewing distance is represented by $d_{v d}$ and the eye's near point by $d_{n p}$. The perceived heights of the object are $h_{v d}$ at the viewing distance and $h_{n p}$ at the eye's near point. Light rays coming from the top of the object in the two positions subtend an angle with respect to the horizontal of $\alpha_{v d}$ (viewing distance position) and $\alpha_{n p}$ (eye's near point). (2) Equivalent result as A1 using an imaginary lens system that produces a virtual image of the object. The object is placed at $d_{n p}$ and the virtual image appears at $d_{v d}$.

\section{PELCO ${ }^{\circ}$ silicon Nitride} \& Silicon Dioxide Membranes

\section{Next Generation SiN TEM Support Films}

- Robust and clean 8, 15, 50 and $200 \mathrm{~nm}$ SiN substrates

-

- EasyGrip ${ }^{\mathrm{T}}$ edges

- Free from debris

- Super flat 8, 15, and 40nm silicon dioxide substrates

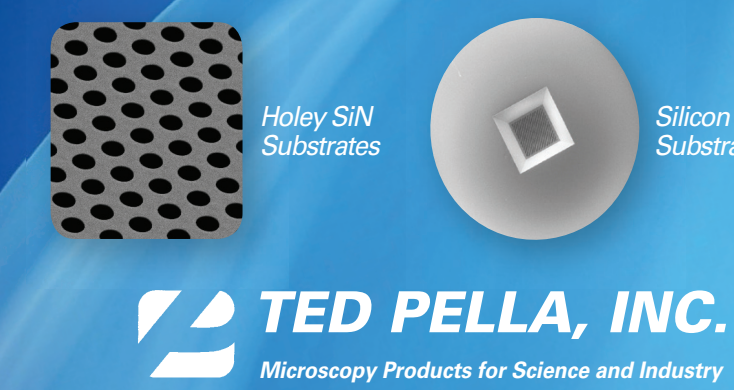

www.tedpella.com sales@tedpella.com 800.237.3526 



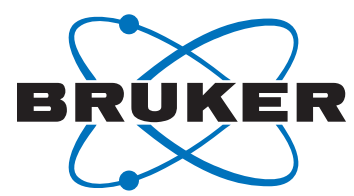

\section{Proven}

\section{Electron Microscopy} Solutions

\section{QUANTAX EDS}

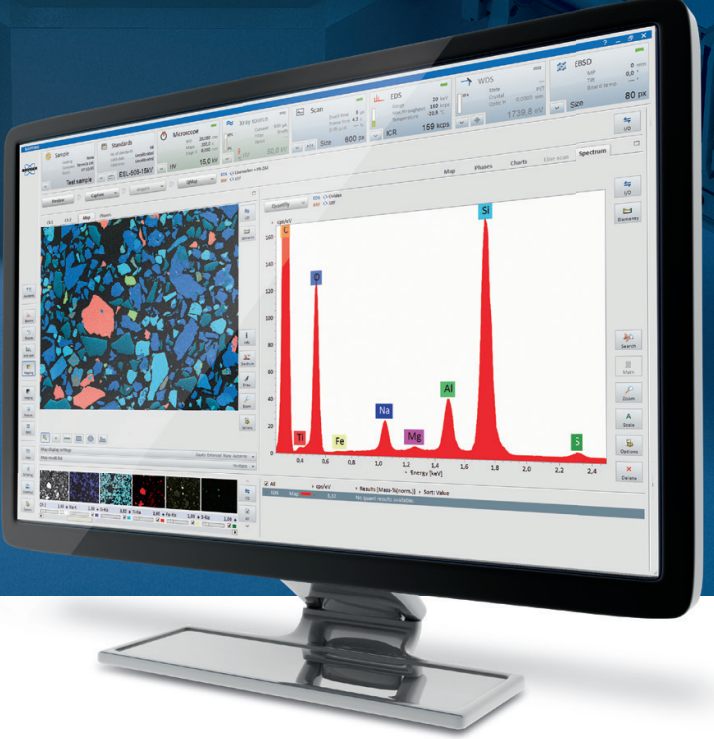

- Featuring XFlash ${ }^{\circledR} 6$ - worldwide leading SDD technology for SEM and TEM

- Delivering the fastest, most accurate EDS results

- Easiest-to-use EDS, available in configurations for every budget

- Integration of EDS, WDS, EBSD and Micro-XRF on SEM under a single user interface

www.bruker.com/quantax

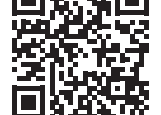

पastin 